\title{
Gaussian-Inspired Auxiliary Non-equilibrium Thermostat (GIANT) for Dissipative Particle Dynamics Simulations
}

\author{
Safa Jamali $^{1^{*}}$, Arman Boromand ${ }^{1 *}$, Shaghayegh Khani ${ }^{1}$ and Joao Maia ${ }^{1}$ \\ 1. Dept. of Macromolecular Sci. and Eng., Case Western Reserve University \\ 2100 Adelbert Road, Cleveland, Ohio 44106 USA \\ E-mail: joao.maia@case.edu
}

\begin{abstract}
We present in this letter an auxiliary thermostat for non-equilibrium simulations in Dissipative Particle Dynamics based on the Gaussian distribution of particle velocities in the fluid. We demonstrate the ability of the thermostat to maintain the temperature under a wide range of shear rates and dissipative parameters, and to extend the shear rate window accessible by DPD significantly. The effect of proposed method on the viscosity of a DPD fluid is studied which is particularly of interest when the rheological behavior of a complex fluids is subject of DPD simulations. Furthermore, performance of the proposed method is compared to the ones from the well-known Lowe-Andersen scheme in regards to temperature and viscosity measurements.
\end{abstract}

\section{Introduction}

Fast-growing computational capabilities associated with increasing interest from scientific community has resulted in emergence of many numerical techniques to study the real-life phenomenon. Perhaps one of the most widely used simulation techniques known is molecular dynamics (MD) which provides atomistic level information about the matter subject to study. However, the length and time scale accessible to atomistic level methods does not allow simulation of phenomena that occur at larger scales. This gave rise to another category of simulation techniques known as mesoscale methods. Dissipative Particle Dynamics (DPD) as a mesoscale method was first proposed in 1992 [1] based on coarse-grained molecular dynamics model. Substitution of hard Lennard-Jones (L-J) potentials in MD by very soft potentials acting on many-atoms ensemble represented by a bead rather than a single atom enables DPD to access length/time scales orders of magnitude higher than the ones approachable by MD. Numerous studies have been performed in order to map the DPD simulation parameters to physically meaningful characteristics [2-6]. Simple formulation and versatility of DPD have attracted many researchers to employ DPD for a wide range of different studies [7-15]. One of the major fields of research in which DPD has been widely

*SJ and $\mathrm{AB}$ have made equal contributions as first authors. 
adapted is fluid dynamics, where rheological features of a fluid are studied at different (equilibrium or under flow) conditions [16-22]. To study non-equilibrium phenomena one would need to assure complete computational stability over the conditions under which the simulation is performed. This includes (but is not limited to) boundary conditions, flow profiles and thermal stability of a system.

In order to study the dynamics of a fluid in response to a specific flow condition, different types of flows can be imposed in the simulation. Similarly to the experimental means of fluid dynamics studies, these flow types include extensional, pressure-driven and drag shear flows. Perhaps the most relevant flow type to real-life phenomena is the drag shear flow, which in its simplest form consists of a steady shear profile of a constant shear rate over the calculation box. Regardless of the flow type, reproducing these flow conditions in the simulation of a particulate system requires use of relevant boundary conditions and often is associated with numerical artifacts. For example, the most widely accepted boundary conditions for steady shear simulation is the well-known Lees-Edwards [23] boundary conditions (LE-BCs). Employing the LE-BCs, one takes into account a series of imaginary cells surrounding the calculation box and imposes the shear profile in the system by simply dragging the upper and lower imaginary cells. However, since DPD utilizes a built-in thermostat that controls the dynamics of a system as well, using LE-BCs in DPD simulations gives rise to two main issues: i) The relative velocity of neighboring particles in the imaginary cells results in a distorted shear profile [24]; ii) At high shear rates the DPD thermostat loses its ability to control the temperature properly. Thus, the temperature deviates from its ideal value, which consequently introduces unphysical behavior in the dynamics of a system [25]. In a recent paper [26], we have addressed the former issue by introducing two different boundary conditions: i) An Eulerian approach which is simply a modified LE-BCs to fit in DPD simulations; ii) A Lagrangian approach that disassembles the shear profile and interaction potentials in one step, and superimposes the shear profile on the calculation box in the second step. The proposed boundary conditions have successfully reproduced imposed shear profiles for a wide range of shear rates and corresponding viscosities were studied by different methods of viscosity measurement.

Different thermostats and alternatives have been proposed in efforts to fix the temperature controlling effect in DPD at rest (equilibrium conditions) [27, 28]. The reason for this is that increasing the time-step in simulation of viscoelastic fluids with DPD even at rest (no-flow) can be problematic [29]. There also have been reports where different measurement techniques or external thermostats have been proposed for DPD simulations under flow conditions [30, 31]. Kremer et al. [32, 33] performed a thorough study on the use of DPD thermostat in non-equilibrium MD simulations, namely on the hard particles rather than usual soft DPD ones, and found DPD as perhaps the ideal thermostat for NEMD simulations as it preserves the hydrodynamics under the flow conditions and it stabilizes the simulations effectively. 
Nevertheless, the latter issue of temperature deviation under high shear rate conditions remains unresolved, as the shear rates subject to previous studies mainly correlate to ones commonly used in MD simulations. The level of shear rates required for simulation of a fluid varies from one to another based on the fluid characteristics and the subject of the study. For instance, in order to study the dynamics of polymer melts under flow, one needs low/intermediate shear rates as this value is strictly dictated by the length scales appropriate for polymer chains [27]. On the other hand, much higher values of shear rates are required when rheology of sheared colloidal suspensions are studied. In fact, since the flow-dependent properties of suspensions such as shear-thickening occur only at shear rates where hydrodynamic interactions dominate the Brownian forces, stable access to a wide range of shear rates is a prerequisite for any simulation technique to capture the flow properties of colloidal dispersions [34]. Thus, here we present a Gaussian inspired auxiliary non-equilibrium thermostat (GIANT) for DPD simulations, enabling of precise control over the temperature. We also compare our results with one of the most widely used thermostats, the Lowe-Andersen [28], and discuss their differences.

\section{Simulation Background}

The equation of motion for a DPD particle is written based on the sum of three main pairwise interaction potentials: A conservative force, $\mathbf{F}_{i j}^{C}$, which gives the chemical identity of a component and the extent of pressure between two particles (equation 1), a random force. $\mathbf{F}_{i j}^{R}$, that introduces thermal fluctuations in a system based on a random component and provides the heat source (equation 2), and a dissipative force. $\mathbf{F}_{i j}^{D}$, which acts against the relative motion of particles and plays the role of viscosity/friction and provides the heat sink (equation 3). Thus, the dissipative and random forces coupled together form the canonical ensemble and act as a built-in thermostat for DPD. One can solve the fluctuation-dissipation equation and define the simulation parameters based on the dimensionless temperature in the system (equation 4).

$$
\begin{aligned}
& \mathbf{F}_{i j}^{C}=a_{m n} w_{i j}^{C} \mathbf{e}_{i j} ; w_{i j}^{C}=\left\{\begin{array}{cc}
\left(1-\frac{r_{i j}}{r_{c}}\right) ; & r_{i j} \leq r_{c}, \\
0 ; & r_{i j} \geq r_{c}
\end{array}\right. \\
& \mathbf{F}_{i j}^{R}=\sigma_{m n} w_{i j}^{R} \frac{\Theta_{i j}}{\sqrt{\Delta t}} \mathbf{e}_{i j} ;\left[w_{i j}^{R}\right]^{2}=w_{i j}^{C}, \\
& \mathbf{F}_{i j}^{D}=-\gamma_{m n} w_{i j}^{D} \mathbf{v}_{i j} \mathbf{e}_{i j} ; w_{i j}^{D}=w_{i j}^{C},
\end{aligned}
$$




$$
\frac{\sigma_{m n}^{2}}{2 \gamma_{m n}}=k_{B} T
$$

When subject to a steady shear profile, as explained before, the average velocity of any given number of particles in a layer (in the velocity gradient direction) must follow the velocity profile of the whole calculation box $\left(\mathbf{u}\left(\mathbf{r}_{i}\right)=\frac{1}{N_{\text {Layer }}} \sum_{i}^{N_{\text {Layer }}} \mathbf{u}_{i}=r_{\text {Layer }} \dot{\gamma}\right.$ where $N_{\text {Layer }}$ is the number of particles in the given layer and $r_{\text {Layer }}$ is the average position of particles in the layer). Having the velocity profile and individual velocities, one can calculate the temperature in the system as given in equation 5 .

$$
T=\frac{1}{3 N} \sum_{i=1}^{N} m_{i}\left(\mathbf{v}_{i}-\mathbf{u}\left(\mathbf{r}_{i}\right)\right)\left(\mathbf{v}_{i}-\mathbf{u}\left(\mathbf{r}_{i}\right)\right)
$$

In this, $\mathbf{u}\left(\mathbf{r}_{i}\right)$ indicates the average velocity of the $i$-th particle's parent layer. Having the layer velocities, one can also calculate the pressure tensor and the shear stress (negative of the $x y$ component of the pressure tensor) and viscosity accordingly based on the expressions given in equation 6 .

$$
\mathbf{P}=\frac{1}{V}\left\{\sum_{i=1}^{N} m_{i}\left(\mathbf{v}_{i}-\mathbf{u}\left(\mathbf{r}_{i}\right)\right) \otimes\left(\mathbf{v}_{i}-\mathbf{u}\left(\mathbf{r}_{i}\right)\right)+\sum_{j>i}^{N} \sum_{i=1}^{N-1} \mathbf{r}_{i j} \otimes \mathbf{F}_{i j}\right\}, \eta=\frac{\left\langle S_{x y}\right\rangle}{\dot{\gamma}},
$$

In practice, DPD simulations of the Groot-Warren water [5] were performed for 3000-24000 particles at the number density of $\rho=3.0$ with a velocity-Verlet time integration scheme and time step size of

$0.01 \sqrt{\frac{m r_{c}^{2}}{k_{B} T}}$ for more than $1.5 \times 10^{6}$ time steps where the first $0.5 \times 10^{6}$ were not included in the statistical averaging, since the system reached its steady state during that time.

\section{Results and Discussion}

Figure 1 shows the temperature as a function of shear rate for: a) a range of dissipative parameters using standard DPD and the time step of 0.01, and b) different time steps. One can argue three main conclusions from the figure 1: i) The temperature deviates from its set value of $k_{B} T=1.0$ in a continuously increasing manner as the shear rate increases; ii) The built-in DPD thermostat loses its capability of holding the temperature as the dissipative parameter decreases, and iii) At the time steps smaller than 0.01, changing the time step size does not affect the temperature of the system as the data points in the figure 1.b fall on the same curve for over two decades of time step sizes. The first two conclusions are expected, because of the nature of the dissipative force. As explained in the previous section, the dissipative force acts against the relative motion of particles and thus increasing the strength of this 
potential retains the velocity of interacting particles in a narrower range. On the other hand, the relation given in equation 4 has to be satisfied and thus, the larger dissipative parameter, $\gamma_{m n}$, the larger the random force controlling parameter, $\sigma_{m n}$; hence increasing the dissipative strength is limited by the noise parameter as well. In contradiction with our results in 1.b, one may expect a better thermostatting effect as the time step size is decreased; however, one should keep in mind that decreasing the time step size directly increases the strength of the random force as it appears in the denominator in equation 2 .
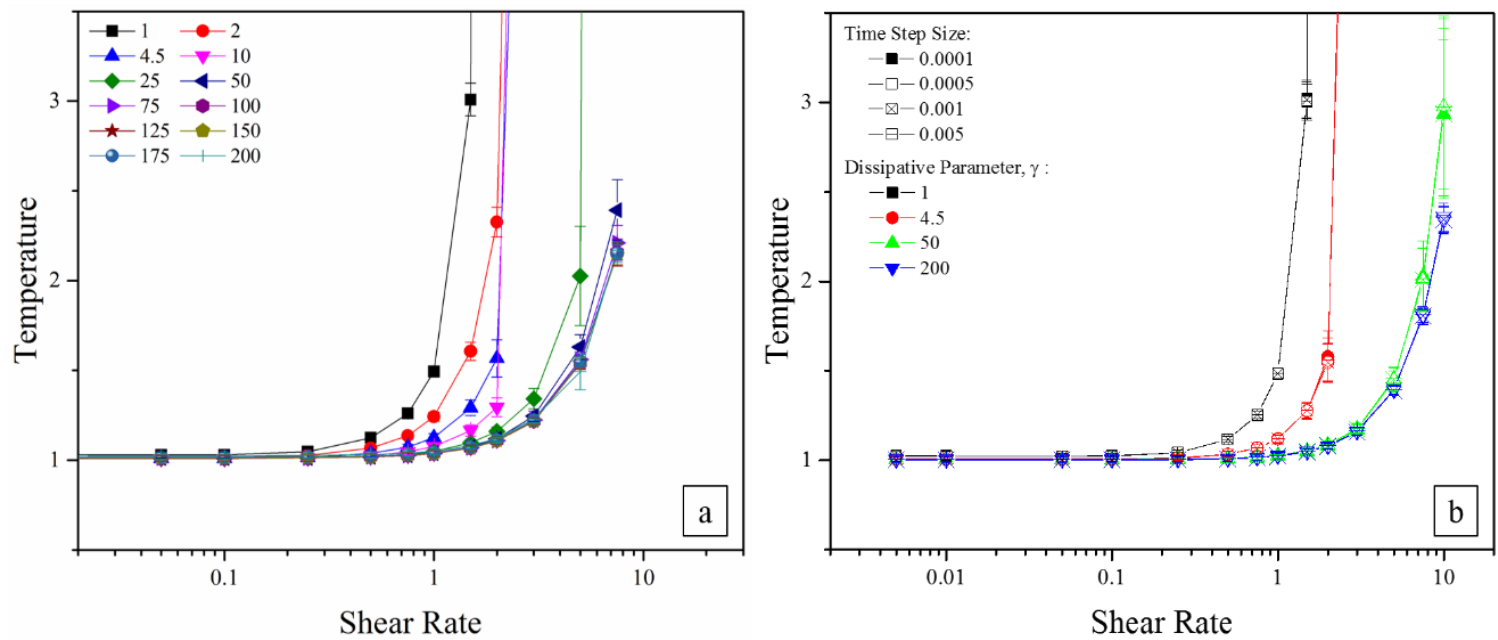

Figure 1. Temperature versus shear rate using DPD thermostat for a range of Dissipative parameters.

In order to better understand the nature of this temperature deviation under high shear rates, one needs to monitor the distribution of particle velocities at each simulation condition. Statistical mechanics dictates that at any given instance the velocity of individual particles of a fluid should form a Gaussian distribution with the maximum at zero and the full width at half-maximum (FWHM) indicating the temperature $[35,36]$. Needless to mention that this has served as the physical foundation of the majority of thermostats used in mesoscale and molecular level simulation techniques [28, 37-39]. This applies to each individual layer of particles subject to shear profile as well, thus one can measure the velocity distribution in the whole calculation box by subtracting the corresponding layer velocities from the particle velocities.

Figure 2 shows the distribution of particle velocities in the flow direction for a range of shear rates and dissipative parameters using the standard DPD thermostat. The velocity distribution curves in figure 2 reveal that regardless of the dissipative parameter used for the simulation, increasing the shear rate broadens the velocity distribution, which shifts the FWHM to higher values and thus higher temperatures in the system are observed; however, increasing the dissipative parameter reduces this effect to some extent. As can be observed in figure 2, it is only at $\gamma=50$ (Fig. 2.d) that the DPD thermostat is capable 
of even keeping the general form of the distribution curves. It should be mentioned that despite the increase in temperature and deviation of the velocities from the expected distribution, the velocity profile in the calculation box remains constant and yields the correct shear rate. This is clear in figure 2.f, where the maximum and minimum velocity of each layer for $\gamma=4.5$ is plotted for a range of different shear rates. The graph shows that as the shear rate increases, the difference between the maximum and minimum velocity of a given layer is increased, meaning that the velocity distribution is broader; however, both maximum and minimum velocities follow the same slope (applied shear rate). In other words, the deviation of the velocity distribution is only a function of shear rate and not the value of the velocity itself, as at the center of the calculation box where velocity is the lowest this deviation is similar to the highest velocity layers. In order to address this issue, we introduced here an external thermostat that acts based on the real-time calculation of the particle velocity distributions in the system. To do this, we have modified the time integration scheme and introduced an additional step where the following occurs:

1- The velocity profile is monitored by dividing the calculation cell into layers,

2- The velocity distribution is formed using the particle velocities in regards to average velocity of the parent layer,

3- FWHM is calculated from distribution curve and,

4- If the kinetic temperature of the fluid has deviated more than $5 \%$ from the set temperature, all the particle velocities are divided by the calculated FWHM in order to make sure that FWHM is corrected to the set value $\left(\mathbf{v}_{i}^{\text {New }}=\mathbf{v}_{i} \frac{k_{B} T}{F W H M}\right)$. 


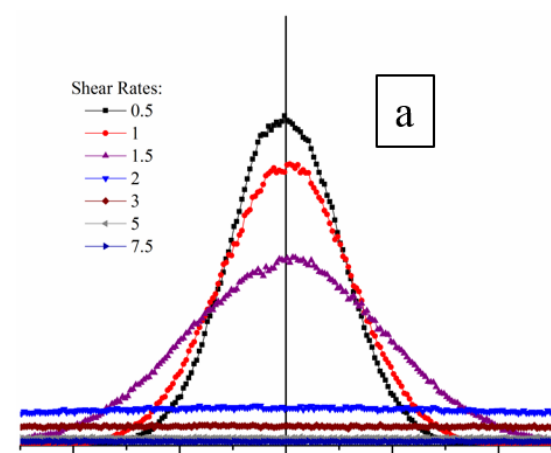

Velocity

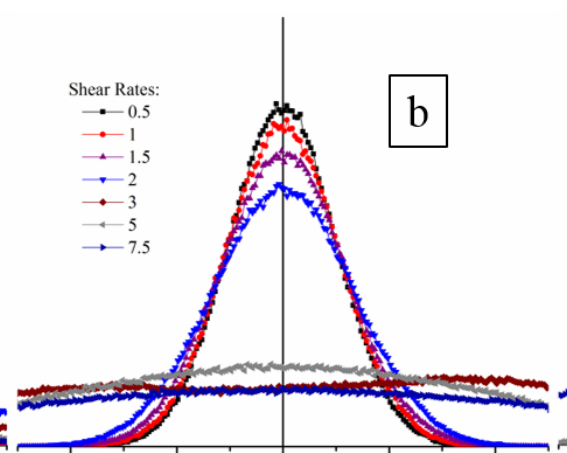

Velocity

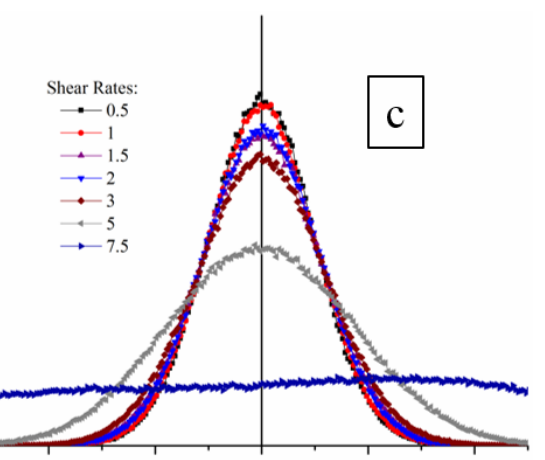

Velocity

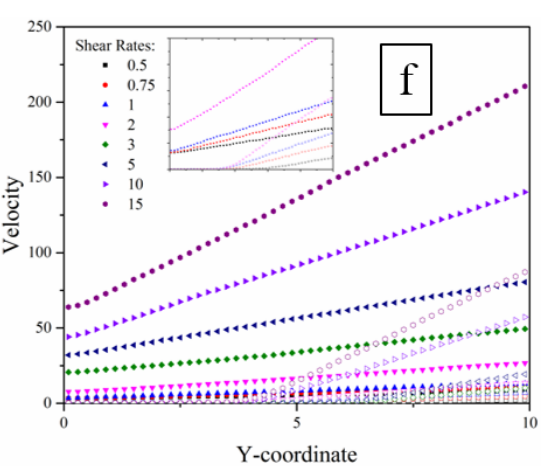

Figure 2. Distribution of particle velocities in the flow direction for different shear rates using different dissipative strength: a) 1, b) 4.5, c) 25, d) 50 and e) 200. f) Maximum (solid) and minimum (empty) velocity profiles of the half calculation box for different shear rates and $\gamma=4.5$. Insert shows different scale of low shear rates for visual purposes.

One should note that calculation of temperature in any DPD simulation, and more pronouncedly in nonequilibrium conditions, is associated with fluctuations in the measured temperature. This fluctuation is normally in the range of less than $3 \%$, meaning that for a system that retains its set temperature of 1.0 , at each individual time step, the temperature might vary between 0.97-1.03 which also has been reported previously $[40,41]$. By imposing an applied shear rate in the calculation box the value of this fluctuation can increase by some extent, while the time-average temperature is kept unchanged. It should be noted that although this slight deviation in the temperature is present, the dynamics of the system is not affected by it, and thus this small deviation can be ignored. The reason for this error is that in order to calculate the temperature, the very initial step is to divide the calculation box into number of layers in the velocity gradient direction and measure the average velocity of each layer $\left(\mathbf{u}\left(r_{i}\right)\right.$ in equation 5$)$. Each of these particle layers take a finite thickness which cannot be too small or too large. A slim choice of layer thickness results in a very few number of particles to calculate the average layer velocity from, while an overestimated layer thickness includes an inherent velocity gradient within itself that gives rise to unrealistic temperature/distribution measurement. In other words, the small deviations in the temperature, 
particularly under shear, are not considered as physical deviations as they are originated by the numerical means of measurement. Hence in applying the thermostat (the $4^{\text {th }}$ step above), two entities (the FWHM and the temperature) being compared are subject to intrinsic numerical errors of the range $<5 \%$ that are of the same nature. The value of $5 \%$ error was employed to ensure that the thermostat is only applied in the system when deviations measured at each time step are larger than the so-called normal deviations measurable in any DPD simulation, and are originated by a physically meaningful change in the velocity distribution function, rather than a common numerical error associated with these types of simulations.

We performed similar simulation studies using the well-known "Lowe-Andersen" [28] scheme coupled with DPD thermostat. This is simply because we aim to study the effect of dissipative parameter on the viscosity of a simple DPD fluid, thus instead of employing a stand-alone LA scheme which excludes the dissipative and random potentials completely, we have coupled this time integration method as an external heat sink with the DPD thermostat. The Lowe-Andersen scheme utilizes the relative velocity if interacting particle pairs as opposed to individual particle velocities. Equation 7-9 shows different steps of the L-A thermostat. In the first step, a relative velocity $\left(\mathbf{v}_{i j}{ }^{\prime}\right)$ is taken from the Maxwellian distribution by using the random number of $\xi_{i j}$ with unit variance and zero mean value. Subsequently, particle velocities are corrected using product of the Maxwellian relative velocity $\left(\mathbf{v}_{i j}{ }^{\prime}\right)$ and the real relative velocity $\left(\mathbf{v}_{i j}\right)$ of particles in the last step. It should be mentioned that this scheme eliminates the random and dissipative forces from the equation of motion, and replaces them by the equations 7-9, and by doing this changes the dynamics of a system abruptly.

$$
\begin{aligned}
& \mathbf{v}_{i j}^{\prime}=\xi_{i j} \sqrt{2 k_{B} T}, \\
& 2 \Delta_{i j}=\mathbf{r}_{i j}\left(\mathbf{v}_{i j}^{\prime}-\mathbf{v}_{i j}\right) \cdot \mathbf{r}_{i j}, \\
& \mathbf{v}_{i}^{\prime}=\mathbf{v}_{i}+\Delta_{i j} ; \mathbf{v}_{j}^{\prime}=\mathbf{v}_{j}-\Delta_{i j},
\end{aligned}
$$

Previously we reported the effect of this scheme on the linear and non-linear viscoelastic properties of the polymer melts at equilibrium and low shear rate out of equilibrium conditions [27]. Figure 3 shows the temperature as a function of shear rate for a wide range of dissipative parameters using: a) our thermostat, and b) the Lowe-Andersen thermostat. Comparing the results in figures 1 and 3 shows that introducing the thermostats provides an excellent control over the temperature at the complete range of shear rates even at the lowest dissipative parameter values of 1 . In the case of the new thermostat, it should be noted that the temperature is stable at the value of 1.06 as opposed to set temperature of 1.0; this constant deviation is attributed to the fact that real-time calculation of the velocity distribution curve and 
consequently FWHM from limited number of velocities (number of particles) is always associated with numerical errors. Also, since finite thickness of each layer is being utilized in order to form the velocity distributions, the FWHM is always calculated for particles belonging to different shear rate regions but same parent layers. This can be decreased by increasing the number of particles, which increases the calculation time. Nonetheless, the introduced thermostat effectively controls the temperature without significant deviations at high shear rates. On the other hand, the LA thermostat shows a contradictory results. Although the temperature remains constant for a wide range of shear rates, it starts to deviate as the shear rate is increased, and more importantly increasing the dissipative parameter results in increased temperatures at high shear rates. As it was explained before, higher dissipative parameters yield narrower velocity distributions, and since the LA scheme is applied based on the relative velocity of interacting particle pairs as opposed to individual particle velocities, increasing the dissipative term reduces the efficiency of the LA thermostat.
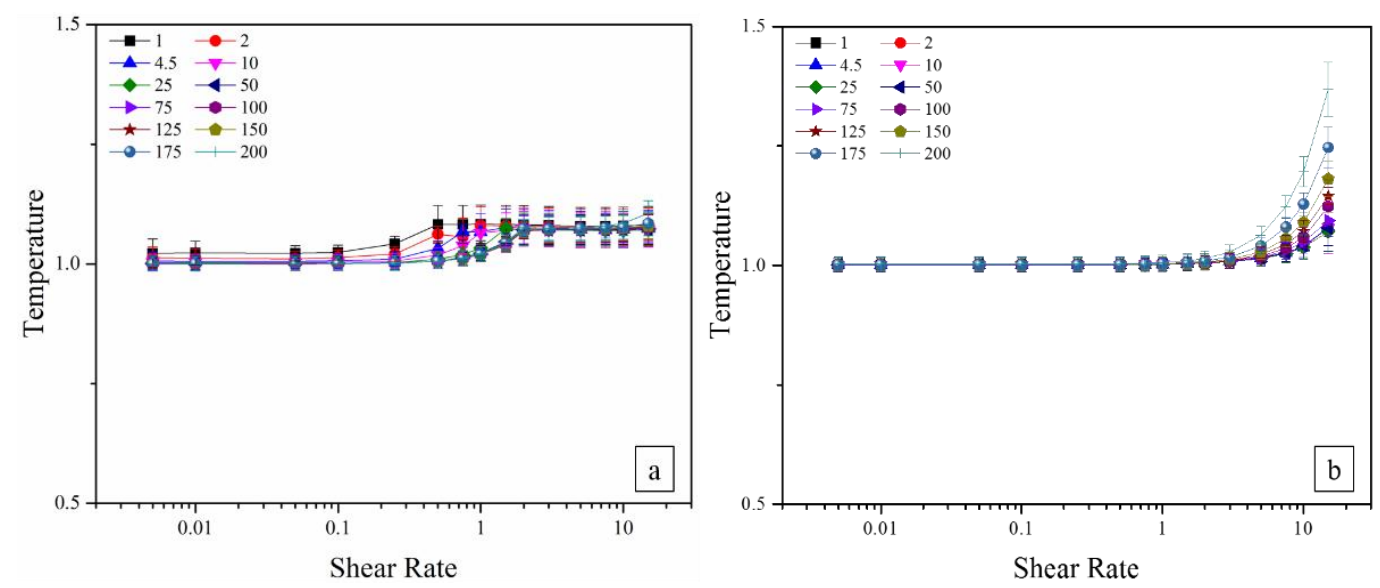

Figure 3. Temperature versus shear rate using: a) our proposed, and b) Lowe-Andersen thermostat.

Figure 4 (a) shows the velocity distribution of the DPD particles at different shear rates using the proposed thermostat and the dissipative parameter of 4.5. Compared to the one obtained from the regular DPD (figure 2.b), one can conclude that the thermostat successfully retains the velocity distributions intact even at the highest shear rates that are not accessible to regular DPD. Also, the velocity profiles presented in figure 4 (b) show that regardless of the shear rate, the maximum and minimum velocity of each layer are in a close vicinity, preventing the distribution to deviate from its proper form. 

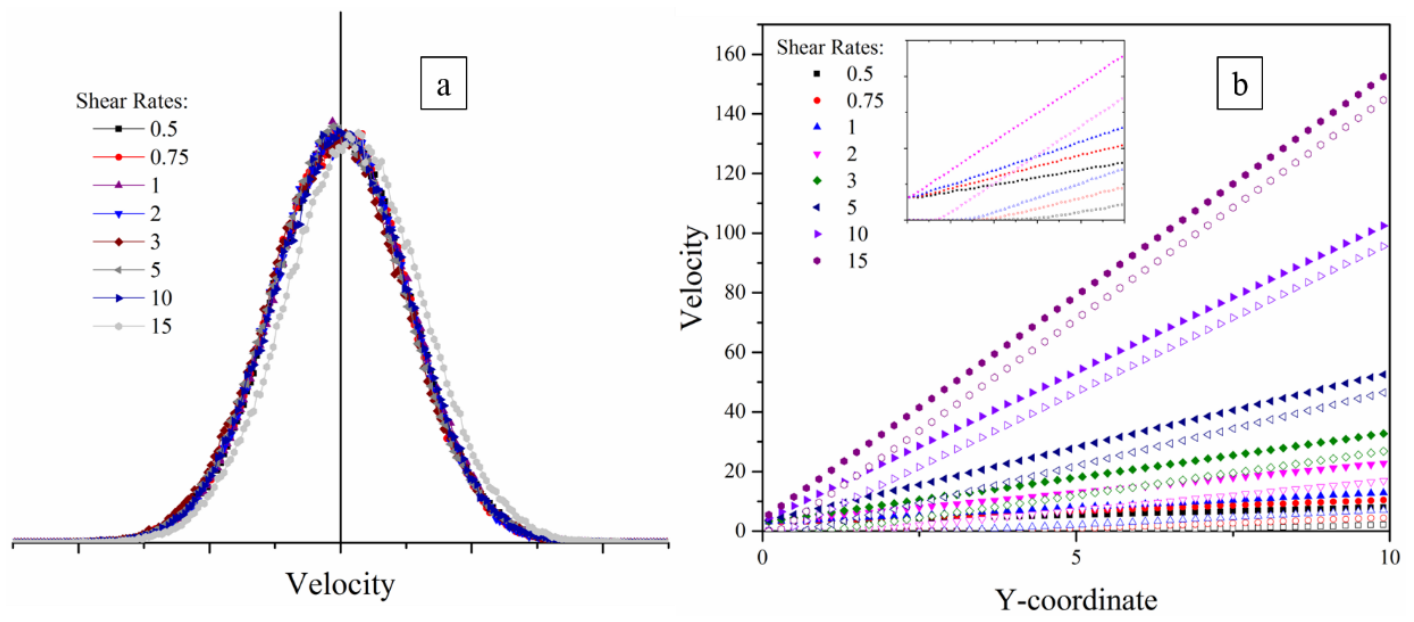

Figure 4. a) Flow velocity distributions for different shear rates. b) Maximum (solid) and minimum (empty) velocity profiles of the half calculation box for different shear rates. Insert shows different scale of low shear rates for visual purposes.

The velocity distribution of a simple fluid under the planar Couette or the steady shear flow can be thought as the velocity of a fluid at equilibrium conditions superimposed by the flow profile exerted by the periodic boundary conditions. A general discussion and the summary of thermostatting methods for flow conditions can be found in Evans and Morris [42]. The velocity of an ensemble of linearly streaming particles at position $\mathrm{r}$ can be written as $\mathbf{u}(\mathbf{r}, t)=\mathbf{i} \dot{\gamma} y$ where $y$ is the position of this ensemble in the flow gradient direction. One can divide a calculation box into several sub-layers and calculate the distribution of particle velocities in each layer. We have plotted this ensemble for the dissipative parameter of 4.5 and the shear rate of 10.0 for the DPD fluid before and after introducing the GIANT thermostat and compared it with the analytical solution of the probability function. The results are presented in figure 5. 


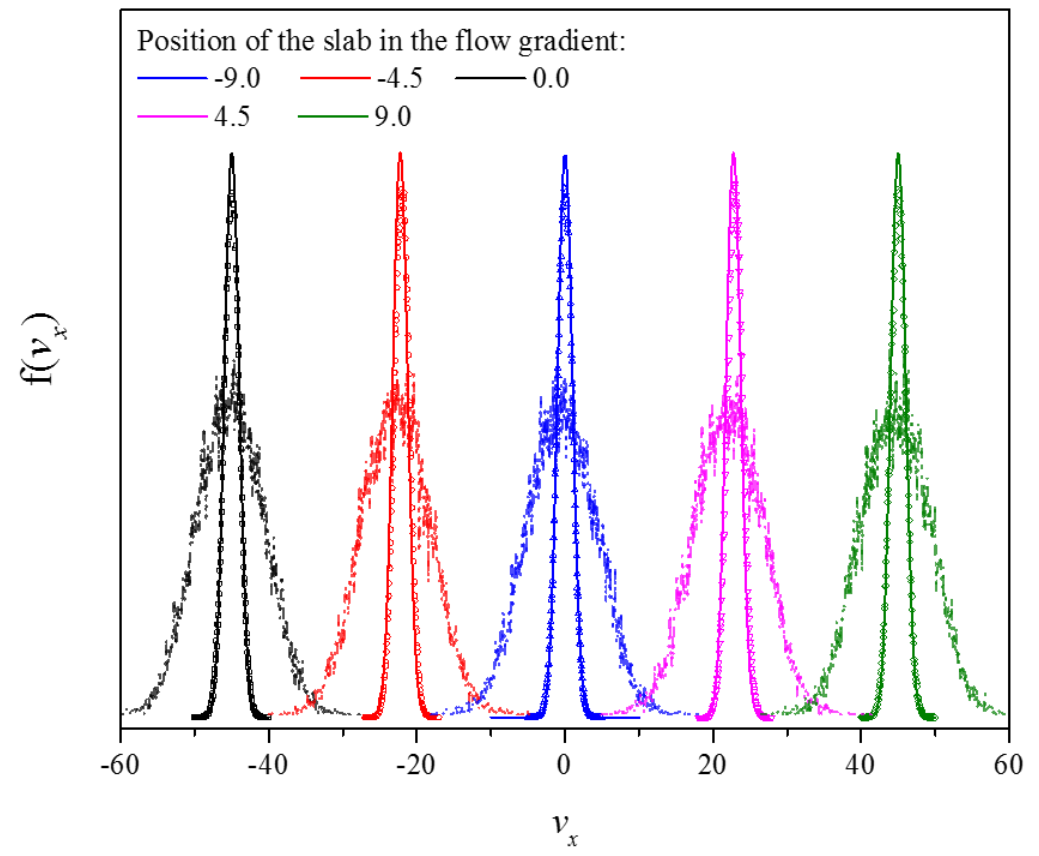

Figure 5. Local velocity distribution functions in different slabs of particle ensembles in the flow gradient direction for standard DPD (dashed lines), GIANT (symbols) and the analytical solution of probability

$$
\text { function } f\left(v_{x}\right)=\sqrt{\frac{m}{2 \pi k_{B} T}} \exp \left(-\frac{m v_{x}^{2}}{2 k_{B} T}\right) \text {. }
$$

The local distribution functions in the figure 5 confirm that the temperature deviations in the standard DPD are direct function of shear rate and not the magnitude of the particle velocities. More importantly, it proves that unlike majority of simplistic velocity rescaling thermostats, GIANT provides a proper statistical distribution both locally and globally. Velocity rescaling techniques have been utilized extensively in Molecular Dynamics studies for decades now. Most well-known and studied algorithms are Andersen [39] and Berendsen [37] thermostats. Although most of these methods are proposed based on the equilibrium conditions, they can be adapted to out of equilibrium and flow simulations with simple modifications [43]. The general approach in these methods can be explained as follows: The kinetic energy of the system is taken at each time step, and individual particle velocities are then rescaled based on the deviation of this kinetic energy from its target value $\left(\alpha=\frac{K}{K_{T}}\right)$. A major shortcoming of these approaches is that poor statistical distributions are generated upon introduction of the thermostat. In order to address this issue different sampling ensembles can be adapted [44]. However, the rescaling procedure used in our method naturally preserves proper statistical distribution. This is because instead of a uniform rescaling factor calculated from the kinetic energy, the deviations of the particle velocities in different directions and the canonical ensemble is taken as the characteristic of the system. Also, since the 
distribution functions are calculated (both locally and globally) only after subtracting the superimposed flow profile from the individual particle velocities, canonical ensembles agree with the analytical solutions of the local distribution functions under flow and in different flow gradient coordinates. By doing this, we ensure that the kinetic energy deviations are stable over time and at different shear rates. Figure 6 shows the normalized kinetic energy (by its target value) over the calculation time for different shear rates for standard DPD and GIANT-coupled simulations.

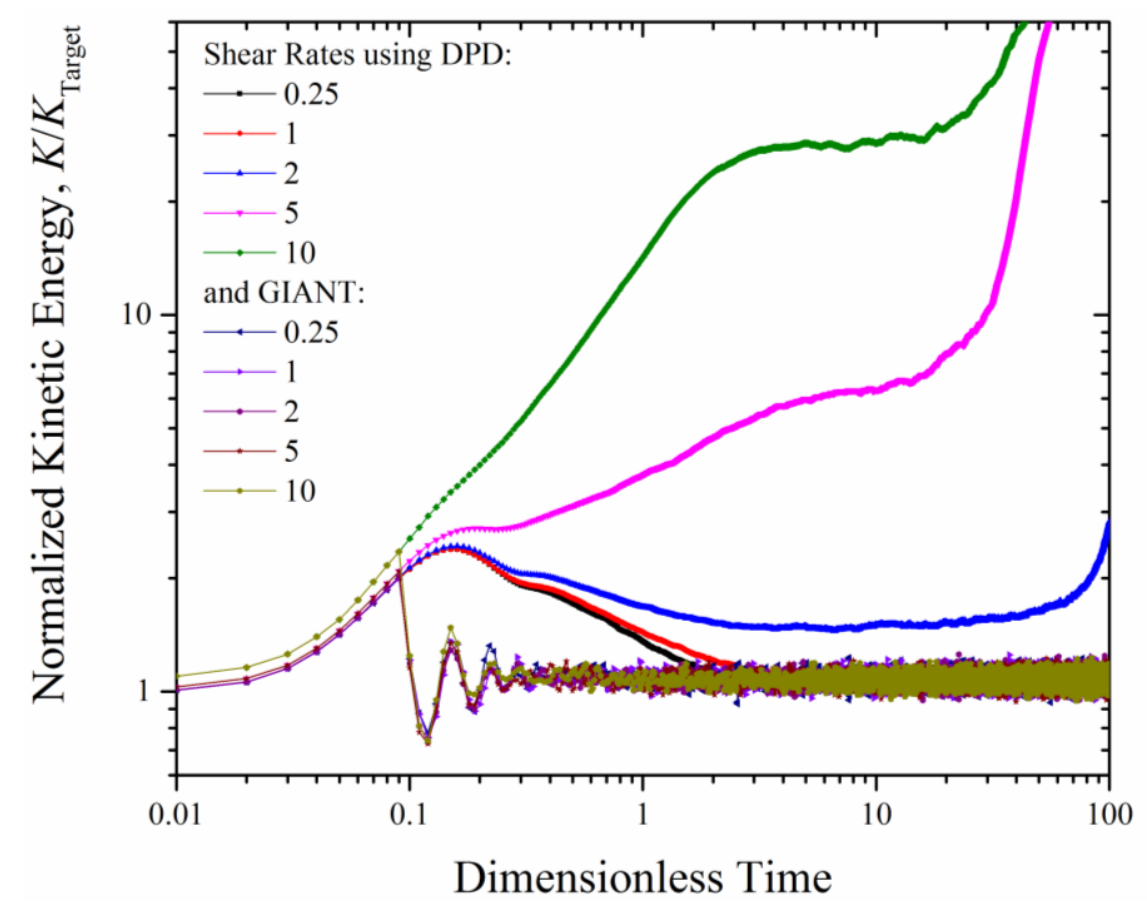

Figure 6. Normalized kinetic energy of DPD fluid at different shear rates as a function of dimensionless calculation time, with and without the new thermostat.

Figure 6 reveals that not only that the deviations of the kinetic energy in standard DPD simulations continuously grow as the simulation time is increased, but also that upon introduction of GIANT the kinetic energy of the system is preserved at all shear rates. It should be mentioned that the initial increase of the energy during the first 10 step is due to the fact that GIANT thermostat is applied after these initial steps required for formation of velocity distributions. One of the main features of DPD simulations is that the momentum is conserved both locally and globally in DPD and thus the hydrodynamic is naturally preserved. The DPD formalism does this via the dissipative and random forces as it continues to do so without the conservative interactions; however, as the distribution curves of the figure 2 suggest, at high shear rates the DPD particles do not represent a physically meaningful fluid anymore and although the momentum is conserved at all conditions, the hydrodynamic is not preserved anymore. Using the new thermostat, one can retain the interacting particles in a realistic velocity neighborhood and thus ensure 
that hydrodynamic is preserved even at high shear rates. This is more pronounces when the relative velocity of interacting particle pairs is studied. Figure 7 shows the relative velocity distribution of interacting particle pairs, for standard DPD and the new thermostat (GIANT), for dissipative parameter of 4.5 and at three different shear rates in different directions.

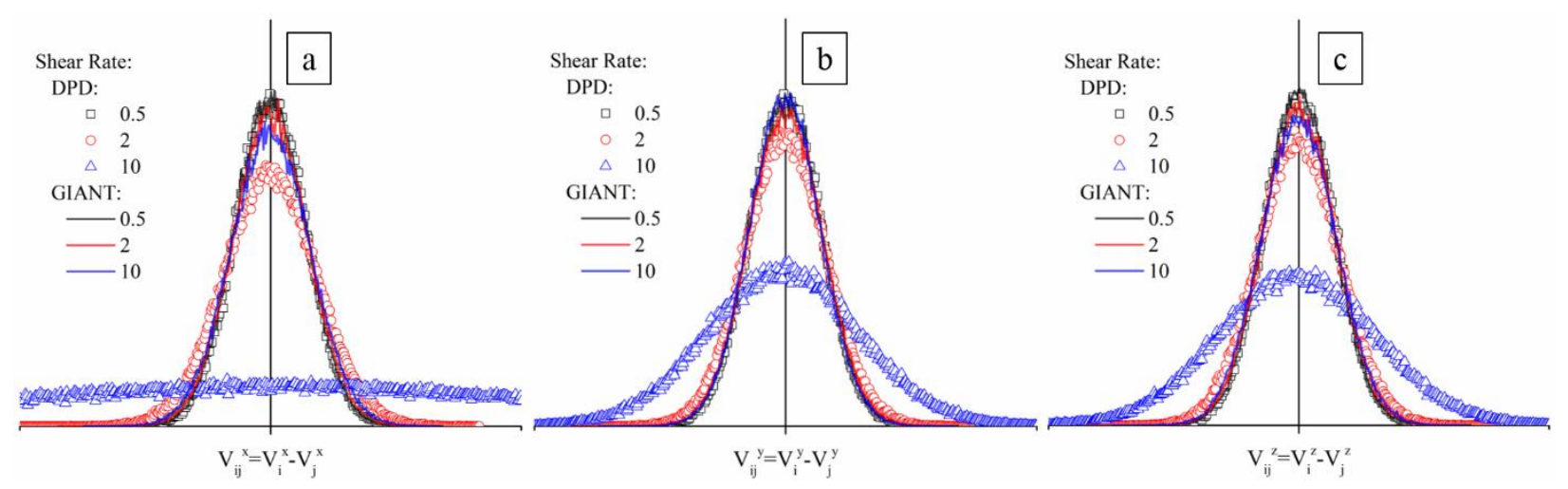

Figure 7. Relative velocity distributions for different shear rates in different directions.

Theoretically, the relative velocity of interacting particles should form a Maxwellian distribution (this in fact serves as the foundation of the LA scheme). At low shear rate of 0.5 , where the standard DPD thermostat successfully controls the temperature, both distribution curves (black line and symbols) fall coincide which proves that at low shear rates the dynamics of DPD remains unchanged upon introduction of the thermostat. Nonetheless, at high shear rates the DPD thermostat shows a broadened distribution which results in abrupt increase of the dissipative stress in the system. At this stage, since the interacting particles hold velocities that largely vary, unrealistic dynamics are recovered. The thermostat however retains the general distribution of relative velocities intact and thus provides proper description of the fluid even at extremely large shear rates.

The physical consequence of the anomalies in standard DPD is clearly observed when transient flow properties of a fluid is considered. Using the L-E boundary condition, at the start-up of the flow particles in the boundary layer are the first ones to adapt the velocities induced by the imposed shear rate. Shortly, the velocity profile is developed in the calculation box by natural diffusion of the momentum through interaction of neighboring particles (dissipative force). This transient flow behavior is characteristic of the fluid and can be studied by monitoring the velocity profile in the flow direction at different simulation times as the system reaches the steady state. Figure 8 shows the velocity profiles of the standard DPD and the ones from thermostat-coupled simulations for the dissipative term of 4.5 and at different shear rates. 

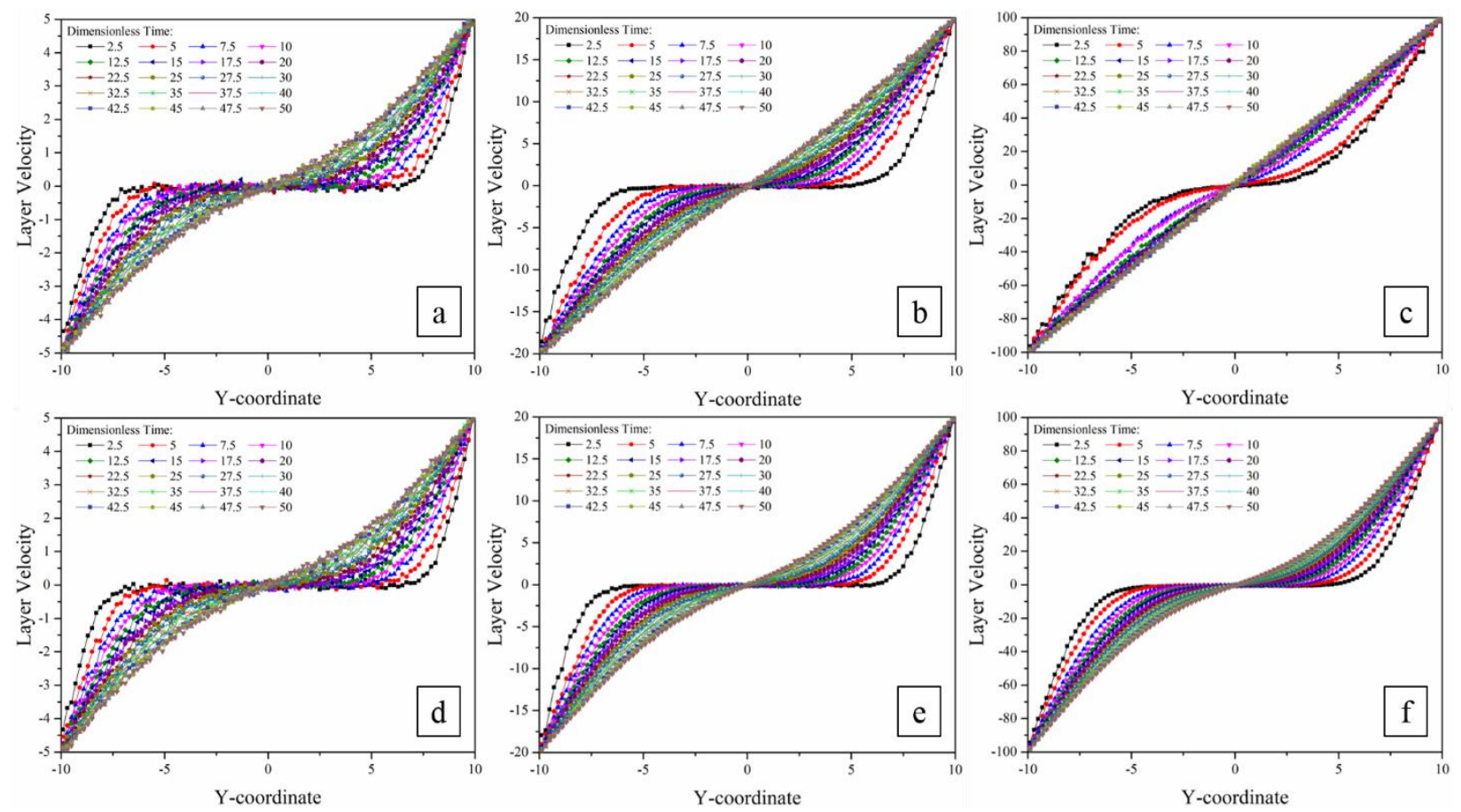

Figure 8.Velocity profile at different simulation times for shear rates of: a, d) $0.5, \mathrm{~b}, \mathrm{c}) 2.0$, c, f) 10.0, using standard DPD (top row) and new thermostat (bottom row).

Comparing the velocity profiles in figure 8.a and d proves that at low shear rates the same transient properties are observed with/out the thermostat. Nevertheless, one clearly observes a change of behavior as the shear rate is increased. The DPD fluid at high shear rates (figure b and c) start to develop the imposed shear profile similar to the ones observed at low shear rates, followed by an unrealistically fast development of the velocity profiles. This change of transient properties is due to an accelerated diffusion of momentum in the system caused by widened relative velocity distributions in figure 7 . The particles that are now in a much wider velocity neighborhood interact through dissipative term, and since the dissipative force spreads the momentum in the system, transient properties of the DPD fluid is significantly affected (unphysical internal momentum source). In contrary, the proposed thermostat provides the proper velocity distributions and relative velocities required for the dissipative force to naturally carry the momentum in the calculation box.

Now with the proper thermostat at hand, we can investigate the rheological response of a DPD fluid to an extended range of shear rates. Figure 9 shows the viscosity as a function of shear rate for a range of dissipative parameters. The flow curves given in figure 9 present the viscosity of the DPD fluid based on two different definitions for the pressure tensor. In general, one can calculate the pressure tensor of an ensemble using equation 6. The first term on the right hand side of equation 6 is the kinetic contribution to the pressure tensor and the second term gives the virial term of the pressure tensor. While one can 
argue that physical definition of the virial pressure requires only the use of conservative force in the virial term [5], there are reports showing the necessity of using all three potentials in the virial term in order to recover the correct rheological response [30,45]. The issue has been studied in detail elsewhere [5], thus here we present our viscosity data measured by taking into account all three types of pair potentials.

The viscosity of the DPD fluid shows a Newtonian plateau at low shear rate regime followed by an unphysical shear-thickening at higher shear rates which is pronounced when low friction parameters are employed. This is a direct consequence of the temperature instability in the simulation, as the kinetic contribution of the stress starts to substantially increase by increasing the temperature. Figure 9.b on the other hand shows that introducing the thermostat effectively increases the range of shear rates by more than a decade. The slight shear-thinning of the viscosity at low $\gamma$ values can be explained by the fact that since the contribution of the conservative force is continuously decreasing (by shear rate), at low dissipative parameter values, the contribution from the random and dissipative potential in stress tensor is not strong enough to compensate for this effect. However, shear-thinning quickly vanishes when $\gamma$ is increased to 25. The results in figure 9.c clearly shows that coupling the DPD thermostat with LA method significantly changes the dynamics of the system. Thus although LA scheme can effectively control the temperature, at the same time it makes the results incomparable to the ones obtain by regular DPD. Obviously, in the case of stand-alone LA time integration scheme, which does not include the dissipative and random force, there is no friction parameter dependence to be studied and the fluid dynamics is solely controlled by the bath probability function introduced in the LA scheme which is studied in depth elsewhere [27].

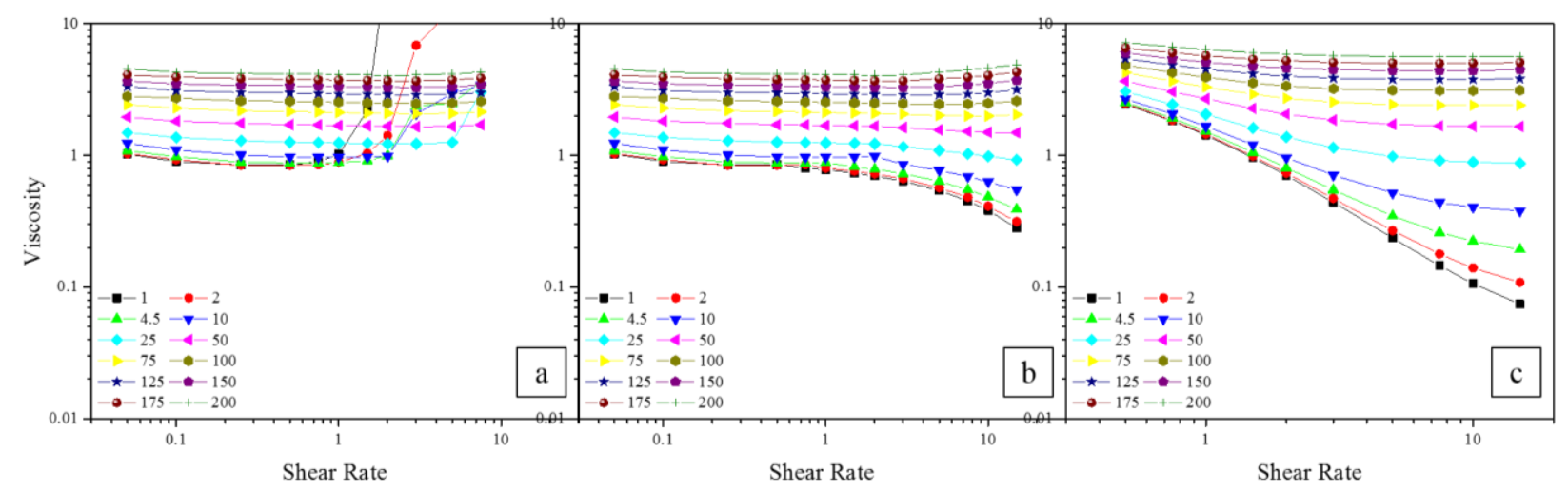

Figure 9. Viscosity vs. shear rate for a range of dissipative parameters using: a) regular DPD thermostat, b) our proposed thermostat, and c) Lowe-Andersen method coupled with DPD.

\section{Conclusions}


In this paper, we present a novel auxiliary thermostat for non-equilibrium Dissipative Particle Dynamics simulations that provides accurate control of the temperature over a wide range of shear rates and dissipative parameters. This was achieved by real-time calculation of the distribution of the particle velocities in regards to their imposed shear profile. Subsequently, the velocity of individual particles was corrected to adapt into the Gaussian distribution of the desired temperature.

In addition to temperature controlling effect of the proposed thermostat, we have investigated the rheological response of the DPD fluid over a range of shear rates in regards to two different definitions

for the stress tensor calculation. Stabilizing the temperature revealed that the shear rate dependence of the viscosity is virtually the same for all the dissipative parameters and only showed slight shear-thinning behavior at low $\gamma$ values and at very high shear rates due to insufficient contribution from the dissipative parameters incapable of compensating for the decrease in the conservative stress.

Thus, it can be concluded that using the proposed approach, the window of shear profiles accessible to DPD simulation can be significantly extended by controlling the temperature without compromising the physical nature of the phenomenon. This can be used in situations where high shear rate conditions are required in order to study a specific phenomenon such as shear-thickening behavior in colloidal suspensions, droplet break-up under flow, and many more.

\section{Acknowledgment}

S.J acknowledges NSF funding through Grant No. CMMI/1068960. A.B acknowledges the NSF Center for Layered Polymeric Systems (CLiPS) for financial support (STC-DMR 0423914). This work was supported in part by an allocation of computing time from Ohio Supercomputer Center (OSC) and High Power Computing Center (HPCC) of Case Western Reserve University.

\section{References}

1. Hoogerbrugge, P.J. and J.M.V.A. Koelman, Simulating Microscopic Hydrodynamic Phenomena with Dissipative Particle Dynamics. Europhysics Letters, 1992. 19(3): p. 155-160.

2. $\quad$ Espanol, P., Fluid particle model. Physical Review E, 1998. 57(3): p. 2930-2948.

3. Espanol, P., Dissipative particle dynamics for a harmonic chain: A first principle derivation. Physical Review E, 1996. 53(2): p. 1572-1578.

4. Espanol, P. and P. Warren, Statistical Mechanics of Dissipative Particle Dynamics. Europhysics Letters, 1995. 30(4): p. 191-196.

5. Groot, R.D. and P.B. Warren, Dissipative particle dynamics: Bridging the gap between atomistic and mesoscopic simulation. Journal of Chemical Physics, 1997. 107(11): p. 4423-4435.

6. Ghoufi, A. and P. Malfreyt, Coarse grained simulations of the electrolytes at the water-air interface from many body Dissipative Particle Dynamics. Journal of Chemical Theory and Computation, 2012. 8: p. 787-791. 
7. Ibergay, C., P. Malfreyt, and D.J. Tildesley, Mesoscale modeling of polyelectrolyte brushes with salt. Journal of Physics Chemistry B, 2010. 114: p. 7274-7285.

8. Malfreyt, P. and D.J. Tildesley, Dissipative Particle Dynamics simulations of grafted polymer chains between two walls. Langmuir, 2000. 16: p. 4732-4740.

9. Schnell, G.M.B., et al., Multiscale modeling approach toward the prediction of viscoelastic properties of polymers. Journal of Chemical Theory and Computation, 2012. 8(11): p. 45704579.

10. Zhao, T., et al., Dissipative particle dynamics simulation of dilute polymer solutions-Inertial effects and hydrodynamic interactions. Journal of Rheology (1978-present), 2014. 58(4): p. 10391058.

11. Mai-Duy, N., N. Phan-Thien, and B.C. Khoo, A numerical study of strongly overdamped Dissipative Particle Dynamics (DPD) systems. Journal of Computational Physics, 2013. 245(0): p. $150-159$.

12. Pan, D., N. Phan-Thien, and B.C. Khoo, Dissipative particle dynamics simulation of droplet suspension in shear flow at low Capillary number. Journal of Non-Newtonian Fluid Mechanics, 2014. 212(0): p. 63-72.

13. Pan, D., et al., Numerical investigations on the compressibility of a DPD fluid. Journal of Computational Physics, 2013. 242(0): p. 196-210.

14. Phan-Thien, N., et al., Exponential-time differencing schemes for low-mass DPD systems. Computer Physics Communications, 2014. 185(1): p. 229-235.

15. Ye, T., et al., Dissipative particle dynamics simulations of deformation and aggregation of healthy and diseased red blood cells in a tube flow. Physics of Fluids (1994-present), 2014. 26(11): p. - .

16. Chatterjee, A. and L.-M. Wu, Predicting rheology of suspensions of spherical and non-spherical particles using dissipative particle dynamics (DPD): methodology and experimental validation. Molecular Simulation, 2008. 34(3): p. 243-250.

17. Fedosov, D.A., G. Em Karniadakis, and B. Caswell, Dissipative particle dynamics simulation of depletion layer and polymer migration in micro- and nanochannels for dilute polymer solutions. The Journal of Chemical Physics, 2008. 128(14): p. -

18. Fedosov, D.A., G.E. Karniadakis, and B. Caswell, Steady shear rheometry of dissipative particle dynamics models of polymer fluids in reverse Poiseuille flow. The Journal of Chemical Physics, 2010. 132(14).

19. Fedosov, D.A., I.V. Pivkin, and G.E. Karniadakis, Velocity limit in DPD simulations of wallbounded flows. Journal of Computational Physics, 2008. 227: p. 2540-2559.

20. Pan, W., B. Caswell, and G.E. Karniadakis, Rheology, microstructure and migration in Brownian colloidal suspensions. Langmuir, 2010. 26(1): p. 133-142.

21. Pivkin, I.V. and G.E. Karniadakis, A new method to impose no-slip boundary conditions in dissipative particle dynamics. Journal of Computational Physics, 2005. 207: p. 114-128.

22. Pivkin, I.V. and G.E. Karniadakis, Coarse-graining limits in open and wall-bounded dissipative particle dynamics systems. Journal of Chemical Physics, 2006. 124.

23. Lees, A.W. and S.F. Edwards, The computer study of transport processes under extreme conditions. Journal of Physics C: Solid State Physics, 1972. 5: p. 1921-1929. 
24. Chatterjee, A., Modification to Lees-Edwards periodic boundary condition for dissipative particle dynamics simulation with high dissipation rates. Molecular Simulation, 2007. 33(15): p. 1233-1236.

25. Pastorino, C., et al., Comparison of dissipative particle dynamics and Langevin thermostats for out-of-equilibrium simulations of polymeric systems. Physical Review E, 2007. 76(2): p. 026706.

26. Boromand, A., S. Jamali, and J.M. Maia, Viscosity measurement techniques in Dissipative Particle Dynamics. Computer Physics Communications, 2015. in press.

27. Khani, S., M. Yamanoi, and J. Maia, The Lowe-Andersen thermostat as an alternative to the dissipative particle dynamics in the mesoscopic simulation of entangled polymers. Journal of Chemical Physics, 2013. 138(17): p. 174903(1-10).

28. Lowe, C.P., An alternative approach to dissipative particle dynamics. Europhysics Letters, 1999. 47(2): p. 145-151.

29. Khani, S., M. Yamanoi, and J. Maia, Mesoscale simulation of entangled polymers: Part II. LoweAndersen thermostat. Bulletin of the American Physical Society, 2014.

30. Marsh, C.A., G. Backx, and M.H. Ernst, Fokker-Planck-Boltzmann equation for dissipative particle dynamics. EPL (Europhysics Letters), 1997. 38(6): p. 411.

31. Whittle, M. and K.P. Travis, Dynamic Simulations of Colloids by Core-Modified Dissipative Particle Dynamics. Journal of Chemical Physics, 2010. 132: p. 124906(1)-124906(16).

32. Praprotnik, M., L. Delle Site, and K. Kremer, Adaptive resolution scheme for efficient hybrid atomistic-mesoscale molecular dynamics simulations of dense liquids. Physical Review E, 2006. 73(6): p. 066701.

33. Soddemann, T., B. Dünweg, and K. Kremer, Dissipative particle dynamics: A useful thermostat for equilibrium and nonequilibrium molecular dynamics simulations. Physical Review E, 2003. 68(4): p. 046702.

34. Jamali, S., M. Yamanoi, and J. Maia, Bridging the gap between microstructure and macroscopic behavior of monodisperse and bimodal colloidal suspensions. Soft Matter, 2013. 9(5): p. 15061515.

35. Kramers, H.A., Brownian motion in a field of force and the diffusion model of chemical reactions. Physica, 1940. 7(4): p. 284-304.

36. Uhlenbeck, G.E. and L.S. Ornstein, On the Theory of the Brownian Motion. Physical Review, 1930. 36(5): p. 823-841.

37. Berendsen, H.J.C., et al., Molecular dynamics with coupling to an external bath. The Journal of Chemical Physics, 1984. 81(8): p. 3684-3690.

38. Nosé, S., A unified formulation of the constant temperature molecular dynamics methods. The Journal of Chemical Physics, 1984. 81(1): p. 511-519.

39. Andersen, H.C., Molecular dynamics simulations at constant pressure and/or temperature. The Journal of Chemical Physics, 1980. 72(4): p. 2384-2393.

40. Travis, K.P., et al., New parametrization method for dissipative particle dynamics. Journal of Chemical Physics, 2007. 127.

41. Travis, K.P., P.J. Daivis, and D.J. Evans, Thermostats for molecular fluids undergoing shear flow: Application to liquid chlorine. Journal of Chemical Physics, 1995. 103(24): p. 1063810651. 
42. Evans, D.J. and G. Morriss, Statistical mechanics of nonequilibrium liquids. 2008: Cambridge University Press.

43. Tuckerman, M.E., et al., Modified nonequilibrium molecular dynamics for fluid flows with energy conservation. The Journal of Chemical Physics, 1997. 106(13): p. 5615-5621.

44. Bussi, G., D. Donadio, and M. Parrinello, Canonical sampling through velocity rescaling. The Journal of Chemical Physics, 2007. 126(1): p. 014101.

45. Marsh, C.A., G. Backx, and M.H. Ernst, Static and dynamic properties of dissipative particle dynamics. Physical Review E, 1997. 56(2): p. 1676-1691. 